\title{
EPOS (EUROPEAN PLATE OBSERVATION SYSTEM)
}

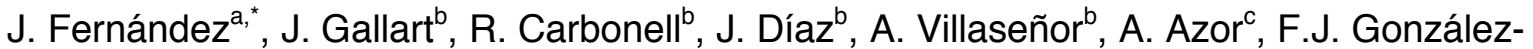 \\ Matesanz $^{\mathrm{d}}$, A. Geyer ${ }^{\mathrm{b}}$, J.J. Curto ${ }^{\mathrm{e}}$, M. Mattesini ${ }^{\mathrm{f}, \mathrm{a}}$, L.R. Rodríguez-Fernández ${ }^{\mathrm{g}}$, J.L. Fernández- \\ Turiel $^{\mathrm{b}}$ \\ ${ }^{a}$ Instituto de Geociencias, CSIC-UCM. Plaza de Ciencias, 3. 28040-Madrid. jft@mat.ucm.es; jose.fernandez@csic.es

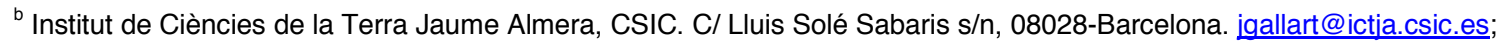 \\ ramon.carbonell@csic.es; idiaz@ictia.csic.es; antonio.villasenor@csic.es; agever@ictja.csic.es; ilfernandez@ija.csic.es \\ ${ }^{c}$ Facultad de Ciencias, Av. de la Fuente Nueva s/n, 18071-Granada. azor@ugr.es . \\ ${ }^{d}$ Instituto Geográfico Nacional, C/General Ibáñez Ibero, 3. 28003-Madrid. fjgmatesanz@fomento.es . \\ e Observatori de l'Ebre, (OE) CSIC - Universitat Ramon Llull, C/ Horta Alta, 38. 43520 Roquetes, Tarragona. jicurto@obsebre.es . \\ ${ }^{\dagger}$ Dpto. de Física de la Tierra, Fac. de Ciencias Físicas. Plaza de Ciencias, 1, 28040-Madrid. mmattesi@ucm.es . \\ ${ }^{9}$ Instituto Geológico y Minero de España, C/ Ríos Rosas, 23, 28003-Madrid. Ir.rodriguez@igme.es .
}

\begin{abstract}
:
EPOS (European Plate Observation System) (https://www.epos-ip.org/) is now established as the only European multidisciplinary and global research infrastructure in Earth Sciences. It integrates several hundred national observatories for the observation and measurement of the internal and dynamic structure of the planet, and in particular in Europe, distributed in 25 European countries, including Spain. The EPOS project, included in the 2008 ESFRI Roadmap, has been recognized by ESFRI in 2016 as a priority project for its implementation, because of its strategic relevance in the European Research Area.
\end{abstract}

Key words: Solid Earth, Geophysics, Geodesy, Geology, Data, Models, Services, Remote Sensing

\section{Resumen:}

EPOS (European Plate Observation System) (https://www.epos-ip.org/) se constituye actualmente como la única Infraestructura europea de Investigación multidisciplinar y global en Ciencias de la Tierra. Integra varios cientos de observatorios nacionales para la observación y medición de la estructura interna y dinámica del planeta, y en particular en Europa, distribuidos en 25 países de Europa, entre ellos España. El proyecto EPOS, incluido en la Hoja de Ruta ESFRI de 2008, ha sido reconocido por ESFRI en 2016 como proyecto prioritario para su implementación, por su relevancia estratégica en el ERA (European Research Area).

Palabras clave: Tierra sólida, Geofísica, Geodesia, Geología, Datos, Modelos, Servicios, Teledetección

\section{Introducción}

El Foro de Estrategia Europea de Infraestructuras de Investigación (ESFRI) (http://www.esfri.eu/) identifica las Infraestructuras de Investigación (RIS por nombre en inglés, Research Infrastructures) que pueden resolver las necesidades paneuropeas a largo plazo de las comunidades europeas de investigación en todas las áreas científicas. La publicación, desde el año 2006, de las hojas de ruta de ESFRI actualizados periódicamente ofrece al Consejo de la Unión Europea una visión coherente y estratégica para garantizar que Europa tenga excelentes $\mathrm{RI}$, accesibles a todos sus investigadores para explotar plenamente su potencial para el progreso científico y la innovación. Estas RIS desempeñan un papel cada vez más importante en el avance del conocimiento y la tecnología, siendo un instrumento clave para reunir la gran diversidad de partes interesadas en obtener soluciones a los problemas que se enfrenta la sociedad hoy en día. Por otra parte, las RIS ayudan a crear un nuevo entorno de investigación en el que todos los investigadores, trabajando tanto en el desarrollo de iniciativas científicas nacionales o multinacionales, tienen acceso compartido a las instalaciones científicas, independientemente de su tipo y ubicación. Las RIS están, por tanto, en el centro del triángulo del conocimiento de la investigación, la educación y la innovación, la producción de conocimiento mediante la investigación, la difusión a través de la educación, y su aplicación a través de la innovación.

EPOS (European Plate Observation System) (https://www.epos-ip.org/) es actualmente la única Infraestructura europea de Investigación multidisciplinar y global en Ciencias de la Tierra. Integra varios cientos de observatorios nacionales para la observación y medición de la estructura interna y dinámica del planeta, y en particular en Europa, distribuidos en 25 países de Europa, entre ellos España. El proyecto EPOS, incluido en la Hoja de Ruta ESFRI de 2008, ha sido reconocido por ESFRI en 2016 como proyecto prioritario para su

*Corresponding Author: José Fernández, jft@mat.ucm.es 
implementación, por su relevancia estratégica en el ERA (European Research Area). Tras la participación de EPOS en la Convocatoria de apoyo a la implementación y operación de proyectos ESFRI, Infradev-3-2015, se obtuvieron los fondos para la fase de implementación actual (EPOS IP) que concluirá en octubre de 2019.

EPOS, es por tanto un plan a largo plazo para facilitar el uso integrado de datos, productos de datos e instalaciones de infraestructuras de investigación distribuidos, para realizar investigación en Ciencias de la Tierra sólida en Europa. EPOS reunirá a científicos de Ciencias de la Tierra, infraestructuras nacionales de investigación, expertos TIC (Tecnología de la Información y las Comunicaciones), responsables de la toma de decisiones y a la población como usuario último, para desarrollar nuevos conceptos y herramientas que permitan dar respuestas precisas, duraderas y sostenibles a las preguntas de la sociedad en temas como los peligros geológicos y los fenómenos geodinámicos (incluyendo geo-recursos) relevantes para el medio ambiente y el bienestar humano. EPOS, mediante la integración de las infraestructuras nacionales y transnacionales de investigación existentes, aumentará el acceso y uso de los datos multidisciplinares registrados por las redes y técnicas de observación de la Tierra sólida, los adquiridos en experimentos de laboratorio y/o producidos por simulaciones computacionales. El establecimiento de EPOS fomentará la interopera-bilidad a nivel europeo y mundial en las Ciencias de la Tierra y servicios a una amplia comunidad de usuarios. Como resultado, miles de investigadores en Ciencias de la Tierra se beneficiarán de los servicios prestados por EPOS fomentando grandes avances en la comprensión de los procesos que ocurren en la dinámica terrestre.

Actualmente estamos próximos a la constitución del Consorcio Europeo de Investigación de la Infraestructura de observación europea de la dinámica del planeta, EPOS ERIC. Desde los comienzos de esta iniciativa europea se ha estado trabajando a nivel español para establecer una plataforma multiinstrumental y pluridisciplinar que aglutine las infraestructuras existentes y actúe de nodo nacional en EPOS. Entre las actuaciones desarrolladas cabe mencionar el proyecto CONSOLIDER 'TOPO-IBERIA'. Este proyecto nace de la voluntad de establecer una plataforma común de trabajo que integre todas las capacidades, bases de datos, software, infraestructuras y equipamientos de los distintos grupos españoles. TOPO-IBERIA cuenta con la participación de 150 investigadores sénior de 10 grupos españoles pertenecientes a 7 universidades (UO; UB; UCM; UAB; UCA; UJ; UGR) y 2 OPIs (CSIC e IGME) y el Real Observatorio de la Armada (ROA).

Los 10 grupos participantes en TOPO-IBERIA han expresado desde el inicio (Convenio de Colaboración) su voluntad de seguir coordinados para el uso y mantenimiento conjunto tanto de la instrumentación como de las BBDD de lberArray una vez finalizado el proyecto. En este sentido destacar que actualmente se está acabando de perfilar un convenio de colaboración para la creación del "Consorcio IberArray" que se prevé firmar a lo largo de 2016. El consorcio IberArray, podría nuclearse como el "nodo" español de EPOS ya que distintas instituciones han mostrado su interés en participar en el consorcio con su equipamiento y bases de datos. De hecho, algunas de ellas están ya operativas en IberArray, como es el caso de las redes sísmicas permanentes, nacionales (IGN) y autonómicas (Andalucía, Catalunya), redes permanentes de GPS, etc.

Por otra parte, hay que destacar que en la fase preparatoria de EPOS, España ha sido el país con mayor número de instituciones que han manifestado explícitamente su interés en formar parte de esta infraestructura. En concreto, unas 25 Instituciones españolas (incluyendo las 10 de IberArray) han explicitado sus equipamientos (instrumentación, personal, costes, etc.), según puede comprobarse en la tabla adjunta y en la web de EPOS (epos-eu.org/ride). EI coste total declarado de estas Infraestructuras españolas de investigación que formarían parte de EPOS es muy remarcable, ya que supera los $45 \mathrm{M} €$.

Por tanto, puede concluirse que España cuenta por primera vez con: (a) Una plataforma multi-instrumental y pluridisciplinar de observación de la Tierra para el estudio del subsuelo y de su impacto ambiental (IberArray). Esta plataforma podría nuclear el "nodo" español de EPOS, ampliando la participación a todas las instituciones españolas que han mostrado su interés por "EPOS"; (b) Una red integrada de centros/grupos bajo el paraguas de una iniciativa europea; (c) Una coordinación a nivel internacional con otros programas similares (p.e., EarthScope); (d) Una activa participación en la fase preparatoria de la gran infraestructura europea EPOS-ESFRI, 25 Instituciones españolas han expresado su interés en las fases subsiguientes, de construcción y operación.

Todo esto ha justificado plenamente la creación de la red Temática EPOS España que apoye el desarrollo de EPOS a nivel europeo, potenciando el papel e importancia de España y sus grupos de investigadores, en el desarrollo e implementación de esta infraestructura, permitiendo, a su vez, configurar un Consorcio Español en Ciencias de la Tierra Sólida en el marco del cual se desarrolle un trabajo científico técnico conjunto y pluridisciplinar de alto nivel. Las infraestructuras de investigación (RIS) que EPOS tiene como objetivo integrar incluyen, entre otros:

- Sistemas geofísicos de observación distribuidos regionalmente (p.e., sismológicos y redes geodésicas).

- Observatorios locales (p.e., geomagnéticos, y observatorios volcanológicos).

- Laboratorios experimentales y analíticos.

- Datos de satélite integrado y la información geológica.

Se ha concedido recientemente una ayuda, en la convocatoria de 2016, para la creación de la Red de Excelencia "EPOS España", coordinada por José Fernández Torres (IGEO, CSIC-UCM) a través del CSIC, (que es el representante nacional actualmente en EPOS, siendo Josep Gallart, ICTJA el coordinador español). La creación de la red EPOS España permitirá apoyar EPOS a nivel europeo, potenciando el papel e importancia de España y sus grupos de investigadores en el desarrollo e implementación de esta 
infraestructura. Permitirá, a su vez, configurar un Consorcio Español en Ciencias de la Tierra en el marco del cual se desarrolle un trabajo científico técnico conjunto y pluridisciplinar de alto nivel.

Para ver la diversidad de las RIS que participan en el plan de integración de EPOS se puede visitar la base de datos de las infraestructuras de investigación para EPOS (RIDE, http://epos-eu.org/ride/), donde actualmente están unos 250 , con más de un petabyte de datos almacenados, más de 70 laboratorios y miles de instrumentos en sísmica, redes GNSS, y observatorios geomagnéticos y volcánicos. Estas RIS institucionales o nacionales existentes en Ciencias de la Tierra sólida en Europa generan datos e información, siendo responsables de la operación de la instrumentación en cada país. Las infraestructuras de investigación constituyen la capa básica del plan de integración de EPOS, que se estructura de la siguiente manera (Fig. 1):
Integrated Core Services (ICS) (Núcleo de Servicios Integrados): proporcionan acceso a los datos multidisciplinares, productos de datos, y datos sintéticos a partir de herramientas de simulación, proceso y visualización. No se trata sólo de acceso a datos: EPOS significa integrar, analizar, comparar, interpretar y presentar datos e información sobre la Tierra sólida. Este es el lugar donde se produce la integración.

Thematic Core Services (TCS) (Núcleo de Servicios Temáticos): son infraestructuras que proporcionan servicios de datos a comunidades específicas (que también pueden ser organizaciones internacionales, tales como ORFEUS en sismología).

National Research Infraestructures (NRI): Las Infraestructuras e Instalaciones Nacionales de Investigación proporcionan servicios a nivel nacional y enviar datos a las infraestructuras europeas de datos temáticas.

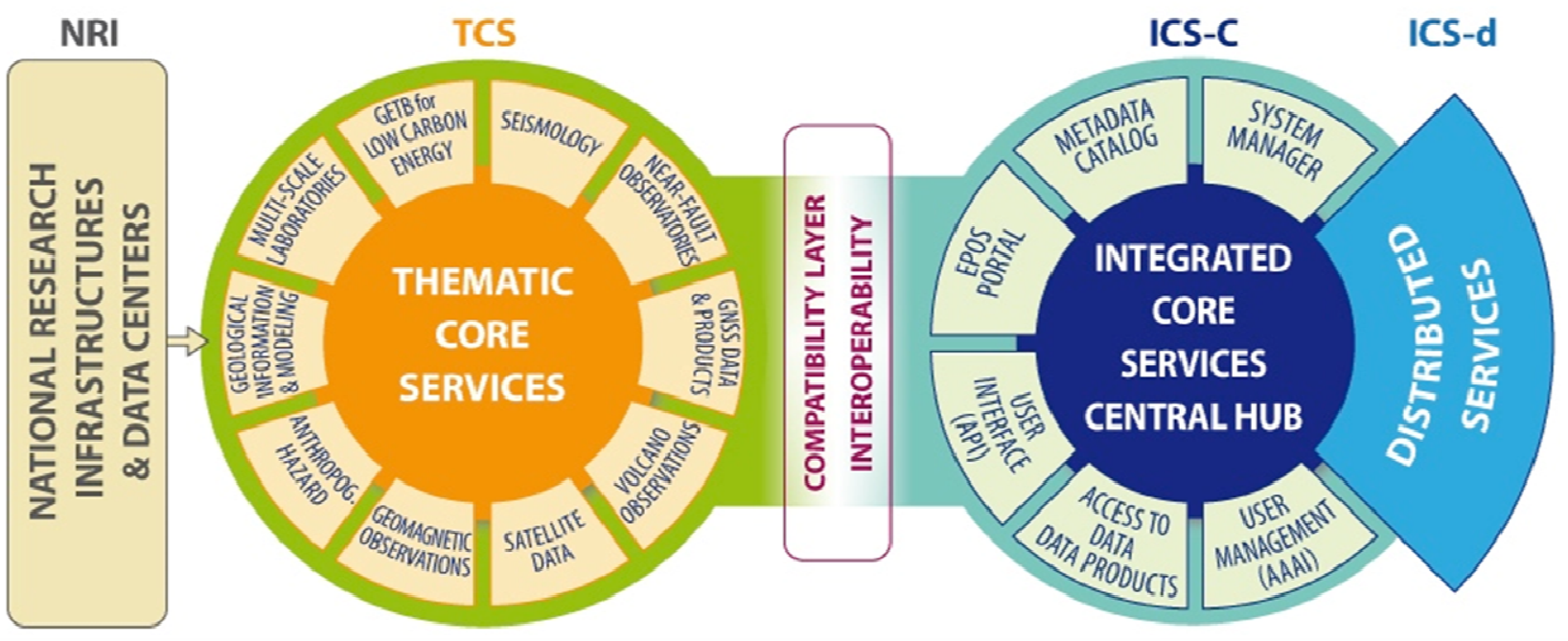

Figura 1: Arquitectura de la infraestructura EPOS.

En EPOS se han determinado 10 servicios temáticos principales, asociados a Work Packages (WP8-WP17). Al configurar la red temática EPOS España se ha considerado como la mejor opción el respetar esta organización, definiéndose los siguientes grupos y coordinadores:

- EPOS España Sismología (WP8), Jordi Díaz Cusí, Antonio Villaseñor (CSIC, ICTJA).

- $\quad$ EPOS España Observatorios Próximos a fallas (WP9), Antonio Azor Pérez (Univ. Granada).

- EPOS España GNSS (WP10), Francisco J. González Matesanz (IGN).

- EPOS España Volcanología (WP11), Adelina Geyer Traver (CSIC, ICTJA).

- EPOS España Espacio (WP12), José Fernández Torres (CSIC, IGEO).

- EPOS España Observaciones Magnéticas (WP13), Juan José Curto Subirats (OE).

- EPOS España Sismicidad Inducida (WP14), Maurizio Mattesini (UCM, IGEO).
- EPOS España Información y Modelado Geológicos (WP15), Luis Roberto Rodríguez Fernández (IGME).

- EPOS España Laboratorios Multiescala (WP16), José Luis Fernandez Turiel (CSIC, ICTJA).

- EPOS España GETB for low carbon energy (WP17) Ramon Carbonell (CSIC, ICTJA).

Veamos los objetivos de cada grupo.

\section{EPOS España Sismología}

El grupo EPOS Sismología España reúne los centros responsables de las diversas redes sísmicas permanentes que operan en nuestro país (IGN, ICGC, IAG-UG, ROA-UCM), así como de los centros que gestionan redes de estaciones sísmicas portátiles (ICTJA-CSIC, IAG-UG, UO, UCM, IGN, ICGC). El grupo participa activamente en el WG8 'Seismology' del proyecto EPOS-IP, siendo una de las entidades que lo forman (CSIC) la responsable dentro de EPOS de la coordinación de las actividades de los parques de estaciones sísmicas portátiles (Mobile Seismic Pools). Parte de los integrantes del grupo participan 
actualmente en la Red de Excelencia 2014 "Red Topolberia-lberarray: Estudios integrados de Geodinámica y estructura de la placa Ibérica" (CGL2014-54582-REDC), vigente en el periodo 20142016 y que a su vez tuvo su origen en el proyecto Topolberia, financiado por la convocatoria 2010 del programa Consolider-Ingenio. En lo referente a las redes permanentes, el principal objetivo que se plantea es la mejora en el intercambio de información en la adquisición, gestión y disponibilidad de los datos obtenidos por las redes sísmicas. Esta labor será de gran importancia para la integración de estos datos en el marco del proyecto EPOS-IP. Respecto a la gestión de las redes sísmicas portátiles, el principal objetivo es establecer un buen mecanismo de coordinación entre los diversos centros, especialmente necesario para dar a conocer la instrumentación disponible y para definir formatos comunes para la distribución de los datos obtenidos y de sus metadatos. Con la constitución de grupo EPOS España Sismología se espera establecer vínculos sólidos de cooperación entre toda la comunidad sismológica del país. La creación de un grupo fuerte y capaz de dar respuesta a los diversos aspectos de la investigación sísmica resulta imprescindible para poder mantener un buen nivel científico internacional.

\section{EPOS España Observatorios Próximos a Fallas}

Los Observatorios próximos a Fallas (NFO) son infraestructuras de investigación multidisciplinares avanzadas basadas en redes integradas de sensores multi-paramétricas que realizan un seguimiento continuo de los procesos físicos y químicos que rigen las fallas activas y la génesis de los terremotos. Estas redes complementan las redes sísmicas y geodésicas regionales con distribuciones de alta densidad de sensores sísmicos, geodésicos, geoquímicos y geofísicos ubicados a corta distancia (hasta 10-20 km) de las fallas activas donde se esperan grandes terremotos.

Los objetivos específicos son participar en el desarrollo y obtención de los objetivos del WP9 de EPOS IP (p.e., adquisición y almacenamiento de series de datos multidisciplinares disponibles, elaboración de normas para el almacenamiento de datos, definición de metadatos, etc...), así como coordinar a nivel nacional la actividad en este campo de científicos de los diferentes campos implicados, fomentar la realización de actividades científicas conjuntas a nivel nacional e internacional, estudiar las posibilidades de creación de NFO en España, su diseño, la instalación y la inclusión en EPOS

\section{EPOS España GNSS}

Las redes GNSS son unas de las herramientas más poderosas en la observación de los movimientos de la placa. Existe una gran heterogeneidad de redes de estaciones permanentes GNSS en España. Por un lado, existen redes GNSS tanto pertenecientes a las administraciones públicas como privadas, que ponen sus datos a disposición libre de los usuarios o no, que su principal objetivo es geodésico/científico o que simplemente su objetivo principal es proporcionar un servicio de correcciones en tiempo real. Además, estas redes pueden tener un ámbito territorial autonómico, nacional o incluso ninguno de los dos anteriores. En principio, todas ellas podrían ser aprovechables para la investigación científica.

Actualmente ya existen organismos de coordinación tanto a nivel europeo, red de estaciones permanentes de EUREF, como a nivel español 'Comisión Española del Sistema Geodésico de Referencia'. De las estaciones GNSS se pueden obtener distintos tipos de datos en función del intervalo de grabación, formato y periodo que abarca. Se considera que el dato útil y suficiente para la observación de la placa europea, que es el objetivo de EPOS, que se obtiene a partir de las estaciones GNSS es el fichero diario con intervalo de grabación de 30 segundos en el formato internacional de intercambio, RINEX. Se pretende colaborar con el resto de grupos para la correlación de los datos GNSS con el resto de observaciones a nivel nacional.

\section{EPOS España Volcanología}

El objetivo principal del WP11 "Volcano Observations" dentro del proyecto EPOS IP consiste en proporcionar, de manera sostenible y a largo plazo, el acceso a datos y productos que se recogen y existen actualmente en los Observatorios Volcanológicos Europeos (VO) y las denominadas Instituciones de Investigación Vulcanológicas (Volcano Research Infrastructures, VRI), que incluyen tanto universidades como centros de investigación que desarrollan una actividad científica de relevancia dentro del ámbito de la volcanología. A nivel europeo, el grupo de trabajo "Volcano Observations" se construye gracias a las infrastructuras operadas por los VOs y VRIs, así como los conjuntos de datos y productos recopilados y desarrollados durante décadas, siendo el objetivo final dentro de EPOS IP, implementar unos servicios que proporcionen y faciliten tanto el acceso a dichos productos y datos como su interoperabilidad. Este es uno de los grupos de trabajo más multidisciplinar de EPOS IP ya que dentro de la larga lista de datos y productos con los que se cuenta se incluyen: (1) Datos y productos de sismología volcánica y GNSS, (2) Datos geológicos, volcanológicos y ambientales, (3) Datos y productos de satélites, (4) Datos y productos de análisis químicos, resultados experimentales y modelos numéricos. Esto conlleva que el WP11 tenga una estrecha relación con el WP8 (Seismology), WP10 (GNSS Data \& Products), WP12 (Satellite Data), WP15 (Geological information and modeling) y WP16 (Multi-scale laboratories). Adicionalmente, el WP11 tiene como objetivo proporcionar acceso a productos y herramientas para la evaluación de la peligrosidad y riesgo volcánico, así como a herramientas tanto experimentales como numéricas enfocadas a la modelización de procesos volcánicos. Por último, se trabaja en el acceso transnacional a recursos e instalaciones para la realización de tareas de investigación básica o durante periodos de reactivación y/o crisis volcánica. A nivel español, los objetivos del grupo EPOS España Volcanología se enmarcan dentro de los descritos para EPOS-IP, habiendo una primera necesidad de identificar y recopilar los datos, productos e incluso servicios ya existentes que puedan ser posteriormente integrados en la infraestructura europea. Se pretende también identificar aquellos recursos e instalaciones que puedan propocionar acceso transnacional a investigadores o 
grupos de investigación tanto nacionales como europeos siguiendo la iniciativa de EPOS IP. Finalmente, se pretende coordinar la comunidad volcanologica en España para fomentar el intercambio de datos y productos, así como para la realización de actividades conjuntas a nivel nacional e internacional.

\section{EPOS España Espacio}

España, en el marco del WP12 de EPOS IP suministrará en abierto a la comunidad científica datos de deformación superficial terrestre determinados mediante interferometría radar de satélite y su combinación con otras técnicas como GNSS, y herramientas de modelado online para su interpretación. La participación de este grupo (en el que se incluyen organismos como CSIC, EEAD, UCM, UPM, Univ. Alicante, IGME, IGC, UPC, IGN, ICGC, CTTC) en la red temática EPOS España permitirá fortalecer las capacidades científicotécnicas de los grupos de investigación en estas técnicas punteras todo el país, que, de forma corordinada explotarán científica y técnicamente el servicio, facilitando sinergias y colaboraciones en el entono EPOS que darán lugar a la generación de conocimientos de frontera y el desarrollo de tecnologías emergentes orientadas a la resolución de las necesidades presentes y futuras de nuestra sociedad. En el marco de la comunidad radar de satélite en España, además de la implementación del Servicio incluido en la solicitud de EPOS IP para el nodo nacional español, se ha iniciado la identificación y consolidación de la comunidad. Esto se está haciendo considerando que EPOS ESPACIO se centra en el estudio de deformaciones superficiales mediante el uso de Interferometría Radar de Satélite, complementando con óptico para desplazamientos horizontales y observación GNSS. Considerando lo reducida que es la comunidad de Gravimetría por Satélite a nivel nacional se ha considerado interesante incluirlos también desde el principio. Se han realizado diferentes reuniones de esta comunidad científico-técnica, donde se han definido los siguientes objetivos: (1) Colaborar en el desarrollo de esta infraestructura; (2) Usarla como marco de colaboración y de crecimiento conjunto; (3) Considerar la solicitud de proyectos de investigación a nivel nacional y europeo para el desarrollo e implementación de esta y otras infraestructuras; (4) Apoyar la creación de un espejo nacional de datos de la ESA suministrándole servicios de valor añadido a través de los servicios implementados en el nodo nacional de Espacio en EPOS IP; (5) Se pretende también colaborar con el resto de grupos, en particular con el WP10 para la fusión de datos GNSS e InSAR a nivel nacional.

\section{EPOS España Observaciones Magnéticas}

El seguimiento de campo geomagnético tiene una larga historia en toda Europa, aunque ha habido poca coordinación a nivel europeo. En EPOS IP, serán objetivos: consolidar la comunidad; modernizar los formatos de archivo y distribución de datos para los servicios existentes, tales como INTERMAGNET; y crear nuevos servicios de datos y modelos geomagnéticos y magnetotelúricos. Los objetivos específicos son: (1) Mejorar los servicios existentes que proporcionan datos geomagnéticos (Red internacional de observatorios magnéticos en tiempo real, INTERMAGNET; Centros Mundiales de Datos de Geomagnetismo, WDC; Monitor Internacionals para efectos geomagnéticos de las auroras, IMAGEN) y servicios que proporcionan los índices geomagnéticos (ISGI-Servicio Internacional de Índices geomagnéticos). (2) Proporcionar a través de una interfaz web información completa de metadados y servicios web relacionados con las bases de datos que se ofrecen. (3) Desarrollar y construir el acceso a datos y modelos magnetotelúricos (MT) que incluye funciones de transferencia (TFS) y series de tiempo (TS), los datos de los MT-arrays temporales portátiles en Europa, y los modelos de conductividad litosféricas derivados de datos TM. (4) Desarrollar puntos comunes de acceso web y bases de datos a los modelos globales y regionales de campo geomagnético interno y externo como el International Geomagnetic Reference Field, IGRF, el World Magnetic Model, WMM, el World Digital Magnetic Anomaly Map, WDMAM, etc., y modelos de conductividad eléctrica. (5) Establecimiento de enlaces entre los distintos Working Packages, WP, a nivel de servicios, productos y modelos. (6) Creación de un nuevo servicio de modelos geomagnéticos basados en datos históricos y arqueomagnéticos.

\section{EPOS España Sismicidad Inducida}

Es indudable la alarma social que causa la ocurrencia de los terremotos y si se trata de terremotos causados de forma no deliberada por el hombre (sismicidad inducida o artificial) es mucho mayor el impacto en la sociedad. En el caso de España existen casos bien documentados de terremotos artificiales o inducidos por presas (Almedra 1972; Tous 1999; Itoiz 2004), Inyección de gas (Cástor 2013), etc. Por tanto, se trata de un riesgo actual y que puede incrementarse en el futuro. Un proyecto como el que se propone permitirá disponer de una amplia base de datos que pueda ser consultada por investigadores y expertos en el futuro. Este grupo se propone los siguientes objetivos: (1) Asistencia y participación en las reuniones del WP14 de EPOS a nivel europeo, apoyando el desarrollo de esta infraestructura. (2) La elaboración de un catálogo de terremotos artificiales o inducidos en España por presas/embalses, inyección de gas o fluidos en la corteza, minas, etc. y que están bien documentados. (3) Recopilación de la documentación ya existente en forma de publicaciones en revistas del $\mathrm{SCl}$, informes, tesinas y tesis, etc. (4) Creación de una base de datos en la nube. En una primera fase esta base de datos estará abierta solo a los investigadores de EPOS. En una segunda fase se discutiría si se pone en abierto y si hay restricciones a los posibles usuarios. En una segunda fase, y en función de la financiación disponible, se podría recolectar datos de los casos estudiados. El tipo de observaciones sería: (a) sismogramas tanto analógicos como digitales; (b) volumen de agua embalsada, tasa de desembalse; (c) volumen de fluido inyectado; (d) sismicidad de la zona; (e) información geológica del emplazamiento; (f) Otros. 


\section{EPOS España Información y Modelado Geológicos}

Los objetivos de este grupo son los siguientes: (1) Asistencia y participación en las reuniones del WP15 de EPOS a nivel europeo, apoyando el desarrollo e implementación de esta infraestructura. (2) Provisión de un acceso eficiente y fácil a los conjuntos de datos científicos del subsuelo obtenidos a partir de estudios de campo y observatorios científicos o por modelado geológico: (i) mapas geológicos en las diversas escalas y detalle; (ii) datos estratigráficos de sondeos y perfotaciones profundas (ICDP/IODP) (iii) bases de datos e inventarios de riesgos geológicos (iv) modelos geológicos (3D y 4D 3D/4D), incluyendo modelos geológicos 3D estructurales, así como los modelos numéricos, los datos también incluyen datos nivel 0 (raw) validados, p. e., perfiles sísmicos. Todos estos datos son de alta calidad y de valor científico único ya que el proceso de modelado es costoso y lento. Para suministrar estos conjuntos de datos se ha ideado una plataforma de gestión de datos basado en modelos de datos común y estandarizada, protocolos y codificaciones, así como en un uso predominante de software libre y de código abierto (FOSS). La interoperabilidad de las aplicaciones por disciplina y dominio depende por tanto en gran medida de la adopción general de las tecnologías y estándar de aceptación (OGC, ISO...) procedentes de esfuerzos realizados en Infraestructura de Datos Espaciales relacionados (p.e., INSPIRE) o de redes ya existentes: OneGeologyEurope, EMODnet-geology o EGDI (European Geologicial Data Infrastructure).

\section{EPOS España Laboratorios Multiescala}

Los objetivos específicos de este grupo coinciden con los de EPOS, desarrollándose a nivel nacional primero, para poder integrarse simultánea o posteriormente en la infraestructura europea, y son:

1. Recoger y armonizar los datos de laboratorio disponibles y emergentes sobre las propiedades y procesos que controlan el comportamiento del sistema de rocas a múltiples escalas para generar productos / servicios accesibles e interoperables.

2. Coordinar el desarrollo, integración y uso de los principales centros y redes de laboratorios en Ciencias de la Tierra sólida.

3. Proporcionar productos y servicios de apoyo a la investigación en Geo-recursos y Geoalmacenamiento, peligros geológicos y la evolución del sistema Tierra.

En el ámbito del paleomagnetismo, este paquete de trabajo se coordinará con la propuesta de la red Maglber que pretende continuar la labor ya iniciada en 2014 de generación de bases de datos paleomagnéticos en Iberia. La red Maglber está coordinada con las acciones ya iniciadas de EPOS-Europa-Laboratorios paleomagnéticos.

\section{EPOS España GETB for low carbon energy}

La energía es una de las temáticas más trascendentales dentro del marco de la investigación en Europa y son muchas las iniciativas dirigidas a incrementar la eficiencia y disminución de los efectos negativos de su producción. Así dentro del Horizonte 2020, uno de los compromisos de la UE es la reducción de las emisiones de gases de efecto invernadero un $20 \%$ por debajo de los niveles de 1990. Este compromiso es uno de los objetivos principales de la estrategia de crecimiento Europa 2020 y está siendo implementado a través de una legislación vinculante. La generación de energía tendrá que desarrollar un esfuerzo en $1+D+i$ temáticas de: reducción de las emisiones (-54 a $68 \%$ en 2030 y 93 a $-99 \%$ en 2050); en la captura y almacenamiento de $\mathrm{CO} 2$; en energías renovables de superficie (eólica, mareomotriz y solar); en temas relacionados con gas de esquisto; la energía nuclear (e. g., gestión de residuos) y geotérmica. Todas estas tecnologías tienen en común que están intensamente relacionadas con el subsuelo (relativamente poco profundo) lo que se denomina capa crítica (subsuelo critico). La zona del subsuelo con la que la sociedad tiene una interacción más intensa. El susbsuelo poco profundo es de gran importancia en muchos campos, desde la ingeniería civil, estudios medioambientales, gestión de residuos, obtención de recursos naturales (agua, hidrocarburos, minerales, etc.). En un continente densamente poblado (EU) se requiere de un alto nivel de gestión del subsuelo crítico y una garantía de la seguridad del medio ambiente en todas las actividades y en particular con las relacionadas con la Energía. En la EU hay una serie de iniciativas (EERA es un ejemplo) involucradas en estos temas. Ademas los países en EU han desarrollado instalaciones e infraestructuras (lo que aquí en EPOS se denomina "Geo-Energy Test Beds") que desarrollan trabajos de investigación, experimentación sobre los procesos (bajo la superficie) que afectan a la sostenibilidad del medio ambiente, incluyendo: el flujo, contención, fuga de fluidos, crecimiento de la fractura hidráulica, hundimientos, sismicidad inducida, las emisiones y tensiones residuales etre otros. En resumen, los GETB son infraestructuras avanzadas/singulares (de investigación) que permiten la comprensión de procesos que hacen posible la sostenibilidad medio ambiental en todos aquellos procesos que impliquen el uso del subsuelo (desde actividades de extracción a actividades de almacenamiento) y son de gran interés para el público en general y para los entes reguladores. Los laboratorios o bancos de pruebas Geo-Energy (Geo Energy Test Beds) son la base de la concepción de los sistemas de gestión de la subsuperficie; y sobre los que se apoya y desarrolla la regulación. Un objetivo clave de EPOS en este paquete de trabajo es el desarrollar sistemas informáticos integrados para facilitar el aceso a la infraestructura de datos generados por estas instalaciones y facilitar el aceso y experimentación en las mismas. Entre otros objetivos está el permitir la publicación en línea de los datos de seguimiento, fomentar la transparencia y mejorar la aceptación del público y la confianza. A largo plazo GETB ayudará el desarrollo de un nuevo modelo de sistema de energía en Europa asegurar: (1) una mayor seguridad e independencia energética, reducir la vulnerabilidad a las 
fluctuaciones del precio de la energía externas; y (2) fomentar el desarrollo de soluciones energéticas europeas. Esta infraestructura integrada trabajará junto con otras infraestructuras de EPOS para crear una plataforma de investigación multidisciplinar eficiente e integral de las ciencias de la Tierra en Europa. Los objetivos específicos son:

- Facilitar el uso integrado de datos, modelos y servicios que hasta ahora han sido operados por separado, en diferentes ámbitos de la geoenergético, para llegar a una comprensión común de los procesos y los recursos energéticos en el subsuelo profundo.

- Proporcionar acceso a la observación, modelado e instalaciones experimentales para mejorar nuestra comprensión fundamental de los entornos geológicos profundos y procesos que regulan la producción y almacenamiento de hidrocarburos a través del tiempo geológico.

- Integrar los datos, los modelos y las instalaciones de GETB dedicado con sistemas geofísicos regionales de observación (redes sismológicas y geodésicas), observatorios locales (incluyendo geomagnéticas y volcán observatorios), laboratorios experimentales y los datos satelitales integradas para obtener el máximo rendimiento de las instalaciones de control existentes no necesariamente dedicado al desarrollo de la energía.

- Promover un enfoque integrado para la planificación de la próxima generación de instalaciones de investigación para geoenergías.

- A través de sistemas informáticos integrados y la entrega a Geo Energía bancos de pruebas, permitir la publicación en línea de los datos de seguimiento para fomentar la transparencia y mejorar la aceptación del público y la confianza.

- En el largo plazo, contribuir a lograr un nuevo sistema de energía en Europa asegurar: (1) una mayor seguridad del suministro de energía, una mayor independencia, y reducir la vulnerabilidad a las fluctuaciones del precio de la energía externas; y (2) fuentes de producción propia de energía que contribuyan a la balanza de pagos, el empleo y los ingresos fiscales.

- Apoyar el diseño de la gestión de los sistemas sostenibles para el subsuelo y desarrollar la regulación para la identificación y explotación de los recursos energéticos geo-subterráneas.

En particular España ha desarrollado iniciativas e infraestructuras muy relevantes centradas en esta temática, por una parte, está la investigación relacionada con la energía y gestión de residuos llevada a cabo de la mano de ENRESA en colaboración con Instituciones de investigación científica (CIEMAT, CSIC, etc.) y Universidades. También hay que subrayar el trabajo desarrollado dentro del proyectos financiados por la UE como el proyecto COMPOSTILLA (http://www. compostillaproject.eu/; https://sequestration.mit.edu/tools /projects/compostilla.html), uno de los programas de investigación punteros en la captura de CO2. Este proyecto junto con financiación nacional a través de CIUDEN (Fundación Ciudad de la Energía) facilitó el desarrollo de instalaciones singulares, laboratorios únicos cuya explotación es de gran valor para los objetivos generales de este "working package de EPOS". Esta red haría posible un primer paso facilitando las tareas de "networking" necesarias para crear un núcleo nacional de instalaciones GETB con un peso específico suficiente para potenciar estas infraestructuras, facilitando datos y acceso a la comunidad científica.

\section{La Red EPOS España}

La creación, y financiación de la red temática EPOS España será una herramienta fundamental para:

1. Resolver un aspecto de gran importancia. Los grupos asociados a los WP 8, 11, 12, 13 y 16, al tener representación en el proyecto EPOS $I P$, disponen de financiación, asociada al desarrollo e implementación de tareas y servicios, mientras los otros no disponen de financiación que permita desplegar la representación española en el desarrollo e implementación de la infraestructura EPOS con relevancia. La constitución de esta red temática permite ayudar a solventar este problema y que España pueda tener un peso en el desarrollo e implementación de esta infraestructura acorde con su importancia científica y económica en Europa.

2. Aumentar las actividades conjuntas de I+D y de difusión a nivel nacional e internacional, aprovechando la colaboración entre los grupos participantes, que incluyen investigadores de múltiples Universidades y Organismos, así como de diferentes CEls (p.e., CEI Campus Moncloa).

3. Facilitar y potenciar la visibilidad internacional de los grupos españoles en EPOS IP, así como conseguir consolidar y aumentar en lo posible el posicionamiento estratégico de los mismos en la implementación de la infraestructura.

4. Fomentar las actividades transversales en el marco de EPOS y surgidos a través del conocimiento mútuo de las actividades desarrolladas.

5. Impulsar la internacionalización de las actividades de I+D en el marco de EPOS, contribuyendo al avance del conocimiento para afrontar los desafíos que la investigación española tiene en el contexto del Espacio Europeo de Investigación.

6. Fortalecer las relaciones con organizaciones internacionales con objetivos convergentes y complementarios (p.e., EuroGeoSurveys).

Se promoverá la comunicación entre los diferentes grupos de investigación mediante la participación y desarrollo de reuniones en el marco de la red temática EPOS España a cuatro niveles: 
a) Participación de los coordinadores de los diferentes grupos en las reuniones de los WP correspondientes del proyecto EPOS IP, informando a los miembros de los grupos españoles de los resultados de las mismas y llevando cuando corresponda la idea propuesta-recomendación del grupo que coordina. Esto permitirá potenciar la participación y visibilidad de los grupos de investigación españoles en la implementación de la infraestructura EPOS.

b) Establecimiento de una página web (https://epos-es.org/).

c) Realización de reuniones de cada grupo de EPOS España y asambleas generales de la red, con presentaciones de resultados, discusiones científicas, propuestas de colaboraciones, etc.

d) Asistencias a reuniones internacionales europeas (EGU y AHPGG) para la participación española en el desarrollo de la Infraestructura durante la fase de implementación y para la difusión del estado de EPOS España.

La futura prosperidad de Europa, y España, en una economía cada vez más competitiva, globalizada y basada en el conocimiento, depende de explotar plenamente el potencial del continente para la innovación científica y tecnológica. La investigación de vanguardia requiere cada vez más inversiones en métodos e instrumentos, y en la informática y datos, que superan la capacidad de cualquier Estado miembro. Esto muestra la necesidad de RIS como EPOS, y la necesidad de participar en ellas de forma importante y visible a través de la red temática EPOS España. EI impacto de EPOS España en la ciencia y la sociedad como red temática en España, y a través de EPOS es claro, y se refleja en: el libre acceso a una infraestructura de investigación multidisciplinar, que apoyará los avances científicos, proporcionando la disponibilidad inmediata y continua de datos de alta calidad y los medios para procesar e interpretarlos; infraestructuras de datos y servicios nacionales y centrales nuevas, contribuyendo a la información, la difusión, la educación y la formación; los planes de implementación, que requieren una inversión estratégica en los RIS a nivel nacional, europeo e internacional obtenido a través de diferentes fuentes de financiación que permitirán actualizar equipos y sistemas; las contribuciones a las actividades de mitigación de desastres y la prevención; colaboraciones con los Campos de Excelencia Internacional, favoreciendo su consolidación y el desarrollo de investigaciones punteras en el área de Ciencias de la Tierra. El desarrollo de la infraestructura EPOS a nivel nacional y europeo producirá una interacción importante con el sector industrial privado. Sirva como ejemplo la interacción ya existente entre el Grupo EPOS España Espacio y el sector industrial, en particular el espacial a través de diferentes empresas con las que se ha colaborado o se está colaborando en Proyectos de investigación de $|+D+|$ tanto a nivel nacional como internacional.

\section{Agradecimientos}

Este trabajo ha sido sufragado por los proyectos EPOS Implementation Phase (EPOS IP) (Grant agreement no: 676564-EPOS IP) del EU VII Framework Program, ESFRI y Red Temática "EPOS ESPAÑA" (CGL201681965-REDT) y Clúster de cálculo y servicio en remoto del nodo español de EPOS Espacio (UCMA15-EE3294), del Ministerio de Economía, Industria y Competitividad, España. 